\title{
A Review on Path Selection and Navigation Approaches Towards an Assisted Mobility of Visually I mpaired People
}

\author{
Waqas Nawaz $^{1 *}$, Kifayat Ullah Khan ${ }^{2}$ and Khalid Bashir ${ }^{1}$ \\ ${ }^{1}$ Department of Computer and Information Systems, Islamic University of Madinah, Madinah, Saudi Arabia. \\ ${ }^{2}$ Intelligent Knowledge Mining and Analytics (IKMA) Lab, Department of Computer Science, National University \\ of Computer and Emerging Sciences, Islamabad, Pakistan. \\ [e-mail: wnawaz@iu.edu.sa, kifayat.alizai@nu.edu.pk, khalid@iu.edu.sa] \\ *Corresponding author: Waqas Nawaz
}

Received February 24, 2020; revised June 19, 2020; accepted July 14, 2020;

published August 31, 2020

\begin{abstract}
Some things come easily to humans, one of them is the ability to navigate around. This capability of navigation suffers significantly in case of partial or complete blindness, restricting life activity. Advances in the technological landscape have given way to new solutions aiding navigation for the visually impaired. In this paper, we analyze the existing works and identify the challenges of path selection, context awareness, obstacle detection/identification and integration of visual and nonvisual information associated with real-time assisted mobility. In the process, we explore machine learning approaches for robotic path planning, multi constrained optimal path computation and sensor based wearable assistive devices for the visually impaired. It is observed that the solution to problem is complex and computationally intensive and significant effort is required towards the development of richer and comfortable paths for safe and smooth navigation of visually impaired people. We cannot overlook to explore more effective strategies of acquiring surrounding information towards autonomous mobility.
\end{abstract}

Keywords: Navigation, Path Planning, Visually Impaired, Information Systems, Obstacle Detection, Autonomous Mobility

This research was funded by Islamic University of Madinah under the grant of first Tamayuz program of academic year 1439-1440 AH and research project number is 24/40. We express our thanks to the administration of FCIS and Research deanship at IUM for their support in every aspect of this work. We would like to acknowledge the work done by all the stakeholders of this project. The content is solely the responsibility of the authors and does not necessarily represent the official views of the Islamic University of Madinah. 


\section{Introduction}

A report by the WHO in 2014 states that there are 253 million Visually Impaired (VI) persons, with 36 Million to be blind, 217 million to have severe visual impairment [1]. Majority of this figure (i.e. around 90\%) belongs to developing countries, so there is a need to develop affordable assistive devices to facilitate smooth navigation of these persons [2]. Digital maps, either proprietary or open-source, are the sources of information for smooth navigation in the real world. These digital maps are self sufficient to fulfill the commute needs of a normal person. However, when it comes to a person with disabilities, these maps become less useful especially for visually impaired persons (VIPs). For instance, navigation paths generated by a general-purpose digital map may not be useful for a VIP and vocal directional information may not be helpful without contextual information, i.e. scene description.

The crowdsourced datasets, e.g. OpenStreetMap (OSM), is one of the prevalent means to easily incorporate customized information for target users to navigate in geophysical space. How effective the crowdsourced datasets like OSM are to provide such specific information for effective navigation of VIPs? We found the answer to this question in [3] where the authors thoroughly study the suitability of OSM for specialized routing services. The authors investigate the completeness of OSM data regarding sidewalk information and concluded that for certain selected cities the available data may suffice the needs of people with limited mobility, however, may not be suitable for other regions of the world. We notice insufficient information of sidewalk data in OSM and reference data (which is manually collected and considered as ground truth to evaluate the effectiveness of OSM data) for Heidelberg, Germany in their statistical comparison. For instance, around twenty-two percent (22.5\%) of sidewalks are available in compared to referenced data, which may result in absence of sidewalk information for most of the users in suburban areas. This shows lack of sufficient information in OSM to be used as it is and requires more efforts to annotate this dataset for more effective utilization in the context of blind people navigation. We can not deny the fact that OSM provides more freedom over proprietary platforms and is a viable choice to develop an effective system for guiding VIPs.

Recent studies depict a comprehensive view on key challenges in accessible maps [4][5]. The authors highlight the current status of available technology and systems for navigation from five different aspects that includes data collection, data management and open standards, modeling needs and abilities, accessible maps and application, and user-centred services. It is found that initiatives have been taken from all aspects, but most of them are in their early stages lacking maturity and completeness. Additional efforts extending existing systems with robust and reliable features or even develop new ones for all aforementioned aspects are required.

\subsection{Challenges in path selection and navigation for visually impaired people}

It is non-trivial to choose a suitable path for visually impaired people to navigate from a single source to one or more destinations. The stakeholders often experience various kinds of challenges while developing navigation solutions for visually impaired people.

- Obstacle Detection and Identification: It is a natural cognitive process for sighted people to inherently detect and identify various kinds of objects during navigation without any assistance. It becomes challenging for a person with visual impairment to utilize other senses and partial available information about surroundings to detect and 
identify objects with limited capabilities. Computer vision and deep learning methods have the potential to provide an assistance and solve these issues to some extent.

- Real-time navigation: A classical navigation problem involves movement of a person from one point to another with continuous guidance, either turn-by-turn or at the abstract level. During navigation, the response time of guiding instructions is critical, as visually impaired may reach road intersection or have an obstacle in front. There are different requirements for navigation in indoor and outdoor environments. GPS is one of the most prevalent means of acquiring location information in an outdoor environment. However, the precision of the acquired location remains an open issue for researchers.

- Contextual Information: Contextual information is very important as it helps us understand the environment. The context is usually determined by collecting relevant information such as distance to the destination, places or landmarks around current location, steepness of the walking path, road crossings, street intersections to name a few. It is a challenging task to acquire vital information about surroundings either directly through content analysis or indirectly from existing repositories.

- Integration of visual and non-visual information: Another challenging aspect in path selection and navigation is to integrate heterogeneous information, which involves visual scenes detected through commodity cameras and non-visual data such as localization with context, to build an understand of the surroundings.

- Availability of autonomous aiding tools and systems: There is a need to provide helping tools and systems to VIPs, in order to assist them in their daily routine commute. One of the key requirements, shared by many practitioners and researchers for those systems and tools, is to provide autonomous assistance from others. The development of aiding tools and systems itself is a complex task due to the limited information, diversity in cognitive abilities, and dynamicity in the surroundings. However, there exists few initiatives in literature with limited functionality to provide reasonable applications and tools for navigation. Making such solutions autonomous enough to make judgements like humans is on top priority, especially in robotics domain.

\subsection{Difference from existing relevant surveys}

We observe similar works in different domains related to this study, however, to the best of our knowledge, there is no existing study on surveying path selection and path navigation techniques in the context of partially sighted and visually impaired people. We discuss existing surveys and studies in the context of their partial relevance to path selection and navigation problem.

Spatial structure and decision-making aspects of pedestrian route selection [6] [7]: It is one of the very early studies on the aspects of spatial structure and decision-making of pedestrian route selection in the urban environment. This study investigates the characteristics of route selection strategies adopted by different types of users from one point of interest to another. The key characteristics affecting the choice of paths includes the count of pedestrians passing through a point of interest (also known as gravity model), safety, greeness, short distance, and quietness. The key findings of this study could only be the initial step to assist in building a better guidance system for general pedestrians, though most of these findings became norms today for an arbitrary path guidance system. However, the behavioral aspects are completely different when we consider pedestrians with visual impairment. 
Machine learning approaches to robotic path-planning [8]: The situation of a visually impaired person seems quite similar to a robot in terms of path guidance as both require assistance to understand the surroundings for mobility. We understand that it is very difficult for a robot to develop an understanding of the real-world surroundings as humans due to lack of senses and cognitive capabilities. The author of this survey discusses various machine learning approach towards robotic path-planning. The relevant aspects of the study for our problem are path planning strategies and machine learning based guidance for robots. The robotic planning is a complex process and involves path planning, inverse kinematics, and reactive planning. It is explained that the machine learning techniques are not suitable for path planning, which is deterministic in nature and other methods have more theoretical guarantee on optimality, completeness and convergence.

Multi-constrained optimal path computation [9]: This work discusses various exact and approximate multi-constrained algorithms to compute optimal paths in computer networks. The multi-constrained optimal path computation is a general problem and can be modeled in other domains such as finding suitable path considering various factors like travel time, distance, safety, etc. The authors state that considering multi-constraints or multiple objectives for finding optimal path is not feasible approach due to underlying complexity of the problem and contradiction among constraints or objective functions. Another brief survey article [10] addresses the multi constrained optimal path selection approaches in Vehicular Ad Hoc Networks (VANET) domain. However, it requires a thorough investigation to determine the suitability of those algorithms in the mobility context of visually impaired people.

Optimal path planning using RRT* based approaches [11]: Rapidly exploring Random Tree Star (RRT*) is a well reputed sampling-based planning approach for robots and UAVs. The basic RRT* algorithm suffers from huge memory consumption due to extensive search space exploration, slow convergence, optimal paths through narrow passages, efficiency of searching strategy, and dealing with physical movement constraints of robots. Therefore, the authors in this article briefly discussed recent developments in this area in terms of variants of RRT* approach to overcome the aforementioned issues via sampling strategies, various kinds of heuristics, multi-directional exploration of the search space, informed or intelligent searching with human input, identifying promising nodes in the search tree, and fixing the number of nodes in a tree for space efficiency. There are open research issues related to finding optimal route for robots with their physical movement constraints and dynamicity in the environment. The formulation of the optimal path planning problem for robots is hard to conceive in the context of visually impaired people due to the complexity of the search space with varying nature of obstacles. We need to understand that the dynamics of the robotic movements and constraints are quite different than human beings.

Navigation systems, Sensor-based and wearable assistive devices for blind and visually impaired people [12] [13] [14]: Recent surveys also enumerate various navigation systems, sensor-based, and wearable assistive devices to provide autonomicity at some extent for blind and visually impaired people in their daily life commute. These studies presented a holistic view and background on the technological advancements, possibilities, and challenges at software and/or hardware level. However, in contrast to these studies, we provide a computation perspective of those technologies and devices for path finding and navigation. We aim to discuss various algorithms and strategies either utilized already or can be useful in path computation and navigation tasks.

In the following sections, we highlight various related studies in reference to navigation assistance to the commuters by explaining each idea briefly. We restrict ourselves or limit our literature review to only studies for visually impaired people to make this study more focused 
in terms of availability of similar techniques and solutions in related domains. The literature review covers mainly path selection approaches (Section 2) considering normal and visual impaired people, path navigation systems, context awareness, visual scene detection for navigation (Section 3). For the sake of completeness, we briefly discuss generic solutions for mobility and the possibilities of utilizing those ideas for visually impaired or low-vision context (Section 4). We also discuss open issues and candidate research directions (Section 5).

\section{Path Selection Approaches}

Path selection is the process of determining a suitable set of sequentially interdependent directional instructions to assist an object movement from one place (source) to another (destination) considering one or more general mobility constraints such as distance, time, and intermediate points of interests (POIs). In other words, it is an approach to compute a set of transition segments starting from a source by following a set of intermediate points to reach a desired destination. The intermediate point refers to either the start or end of a transition segment. A transition segment has an arbitrary length with associated travel time and POIs. The terms path and route are interchangeably used in this study. We present general approaches prior to the one specific for visually impaired to have an understanding of the literature. Below we present a formal definition for path selection problem so that we can learn about existing works more accurately.

Definition 1: Path Selection Given a source s and destination d, our first priority is to compute possible paths between $\mathrm{s}$ and d, i.e. $\mathrm{P}=\left\{\mathrm{p}_{0}, \mathrm{p}_{1}, \ldots \mathrm{p}_{\mathrm{i}}, \ldots \mathrm{p}_{\mathrm{n}}\right\}$, where $\mathrm{p}_{\mathrm{i}} \in \mathrm{P}$, and $\mathrm{p}_{\mathrm{i}}=\left\{\mathrm{seg}_{0}=\mathrm{s}, \mathrm{seg}_{1}, \ldots\right.$ seg $_{\mathrm{i}}$, ...seg $\left.\mathrm{m}_{\mathrm{m}}=\mathrm{d}\right\}$. Having obtained a set of paths $\mathrm{P}$, we then aim to filter out a path pjthat satisfies the given $\mathrm{k}$ global constraints, i.e. $\mathrm{k}=\left\{\right.$ const $_{0}$, const ${ }_{1}, \ldots$ const $_{\mathrm{i}}, \ldots$ const $\left._{\mathrm{k}}\right\}$. The global constraints refer to the requirements of a person like visit to different types of venues before reaching the destination.

\subsection{General Techniques for Route Selection}

In this section, we review path or route selection techniques that are not specific to the movements of VIPs. Our motivation, to review these general-purpose approaches, is to broaden the horizon of the readers and provide better understanding of the problem.

\subsubsection{Skyline query-based path selection}

In order to determine a route from one point to another, at the beginning of each transition segment we may have multiple possibilities to choose the next transition segment, which result in multiple paths for a pair of points. Skyline queries are used in multi-criteria optimization/decision problems. Given a set of points (or paths) $\mathrm{p}_{1}, \mathrm{p}_{2}, \ldots, \mathrm{p}_{\mathrm{i}}, \ldots, \mathrm{p}_{\mathrm{n}}$ the skyline query returns a set of points $\mathrm{P}$, called skyline points, such that any point $\mathrm{p}_{\mathrm{i}} \square \mathrm{P}$ is not dominated by any other point in the dataset. The aim of a skyline query is to find a solution which (a) satisfies certain number of requirements and (b) must provide superior results compared to other set of contemporary solutions [15]. We find that a skyline query has a number of applications in various domains like friend recommendation in location based social networks [16], road networks [17], and path planning [18] among others. Comparison of skyline-based approaches is presented in Table 1.

In the case of friend recommendation, the authors in [16] propose a system of recommending friends using multiple criterion giving rise to multiple recommendations. The authors use skyline query to restrict the candidate size of recommendations. In [17] [18], the authors 
formulate the path selection problem in road networks as the skyline path discovery. When a path is not dominated by any other path within a given pair of points, i.e. source and destination, for multiple path search criteria (such as shortest distance, minimal traffic flow, less travel time, fewer toll tax points and number of traffic lights, less fuel consumption to name a few) is considered as skyline path. The authors record that many online location-based services such as Bing Maps and Google Maps provide optimal paths between a given source and destination, however, they consider single criterion for path recommendation. Hence, considering a skyline path which satisfies multiple constraints is always useful.

The authors in [19] present an online algorithm for skyline path computation. Their approach continuously produces results during the execution of the algorithm and yields more results along the continuation. Such an approach facilitates the user to interactively operate the system by changing preferences, which is not possible in existing batch-oriented approaches for skyline path computation. The key idea of their algorithm is to use nearest neighbor (NN) search process with skyline path computation. The NN searching algorithm determines a set of closest points to a reference point, e.g. an origin $(x, y) \rightarrow(0,0)$ in a $x-y$ plane, where we have set of two-dimensional points. Similarly, when we determine a non-dominating path over two dimensions, e.g. distance and time, by minimizing the distance and time values, which is equivalent to find paths closer to the origin. Continuing the success of using NN search, the authors in [20] further make use of it to overcome the shortcomings such as multiple accesses of the same node in the underlying index structure and large space overhead. SaLSa [21] presents another efficient skyline computation approach which pre-sorts the input relational data before applying the dominance test. In particular, the SaLSa uses sorting to stop further fetching of rows from a data table or input stream in order to limit the number of rows to work upon. In this way, sorting function helps that all the tuples which does not satisfy the given criteria are dominated by already computed tuples.

Table 1. This table summarizes various skyline query based approaches in terms of information utilized, the computation strategy, distance measure, application domain.

\begin{tabular}{lllll} 
Articles & $\begin{array}{l}\text { Necessary information } \\
\text { used for Algorithm } \\
\text { Execution }\end{array}$ & $\begin{array}{l}\text { Skyline computation } \\
\text { strategy }\end{array}$ & $\begin{array}{l}\text { Distance } \\
\text { measure }\end{array}$ & $\begin{array}{l}\text { Application } \\
\text { Domain }\end{array}$ \\
$\begin{array}{l}\text { Friend } \\
\begin{array}{l}\text { Recommendation } \\
\text { by Using Skyline } \\
\text { Query [16] }\end{array}\end{array}$ & $\begin{array}{l}\text { information using } \\
\text { location-based social } \\
\text { networks }\end{array}$ & $\begin{array}{l}\text { Divide and Conquer, } \\
\text { Block-Nested Loop } \\
\text { based or } \\
\text { nearest-neighbor based }\end{array}$ & Shortest Path & $\begin{array}{l}\text { Social } \\
\text { Networks }\end{array}$ \\
\hline $\begin{array}{l}\text { Shooting stars in } \\
\text { the sky: an online } \\
\text { algorithm for } \\
\text { skyline queries } \\
\text { [19] }\end{array}$ & $\begin{array}{l}\text { Index structure of the data } \\
\text { to facilitate computation }\end{array}$ & $\begin{array}{l}\text { Nearest Neighbor } \\
\text { Approach }\end{array}$ & Euclidean & General \\
\hline $\begin{array}{l}\text { An optimal and } \\
\text { progressive } \\
\text { algorithm for } \\
\text { skyline queries } \\
\text { [20] }\end{array}$ & $\begin{array}{l}\text { Index structure of the data } \\
\text { to facilitate computation }\end{array}$ & $\begin{array}{l}\text { Nearest Neighbor } \\
\text { Approach }\end{array}$ & Euclidean & Databases \\
\hline $\begin{array}{l}\text { Finding Skyline } \\
\text { Paths in Road } \\
\text { Networks [17] }\end{array}$ & $\begin{array}{l}\text { Path segments on road } \\
\text { network }\end{array}$ & Path Dominance Test & Shortest path & Road \\
\hline $\begin{array}{l}\text { Route skyline } \\
\text { queries [18] }\end{array}$ & $\begin{array}{l}\text { Models the road network } \\
\text { as a multi-attribute graph }\end{array}$ & $\begin{array}{l}\text { Graph Embedding } \\
\text { Approach }\end{array}$ & Databases \\
\hline
\end{tabular}




$\begin{array}{llll}\begin{array}{l}\text { SaLSa: } \\ \text { computing the } \\ \text { skyline without }\end{array} & \begin{array}{l}\text { Existing sort mechanism } \\ \text { in SQL }\end{array} & \begin{array}{l}\text { Block-Nested Loop } \\ \text { Approach }\end{array} & \begin{array}{l}\text { Lexicographic } \\ \text { sorting of the } \\ \text { data points }\end{array} \\ \begin{array}{l}\text { scanning the } \\ \text { whole sky [21] }\end{array} & & \end{array}$

Summary: Considering the aforementioned and versatile applicability, the skyline query can also be utilized for planning a VIP navigation path. For instance, given the current location and the destination, the system may recommend a multi-criteria path to a VIP that avoids movement on a heavy traffic street, has proper footpaths, and no market in between. The response time of the majority skyline path computation strategies is not realistic for VIP real-time scenarios, therefore difficult to adopt as it is and require thorough investigation.

\subsubsection{Heuristics and Al-based path computation}

The path optimization problem has a long history and robust approaches have been developed in the literature. Heuristics and artificial intelligence techniques are prevalent in exploring the search spaces and optimal path discovery problems. In this section, we will explore some representative studies of this domain towards path computation. We provide an overview of the heuristics and AI-based approaches in Table 2.

Ants have limited resources for smooth navigation and rely on indirect form of communication with other ants using a chemical called pheromone. On its way towards food sources from the nest, an ant leaves pheromone on the passage which serves as hint for other ants. In this way, movement trails are established, and the most frequently used trails are developed as common paths, whereas the least frequent ones vanish with the passage of time. This process can also be mapped to the creation of trails by human pedestrians in deserts and mountains, which become permanent when used or followed repeatedly. These repetitive following of the trails actually refer to the concept of reinforcement random walk. In [22] [23], the authors mimic the movement of ants from the nest to the source of food to the concept of "reinforced random walk". In particular, the authors in [22] use these concepts in the domain of electronic networks whereas [23] targets the domain of graph theory. Random walk-based solution has also been utilized to identify the candidate genes linked to certain types of diseases in the domain of biomedical sciences [24].

There exist a number of approaches for path selection and computation of a robot [25] [26] [27] [28], which are useful as both humans and robots lack visual perception. Robots have limited resources to find an obstacle-free and optimal path from a given source to the destination. The authors in [25] propose a hybrid approach for path selection using Cuckoo Search and Bat algorithm to find a global optimum solution. They first identify a local optimum solution using Cuckoo Search Algorithm, which becomes an input to Bat Algorithm to find a global optimum solution. In this way, a combination of both techniques enables discovery of an optimal path for the robot. Similarly, the authors in [26] use A* algorithm to plan a path for the robot. In the given scenario, $A^{*}$ algorithm operates in the space represented in the form of grid. The movement is possible in 8 possible directions in a grid space; hence the robot has to evaluate each neighboring grid cell. In order to overcome this shortcoming, the authors make use of modified A*, i.e. Basic Theta* and Phi*. In Basic Theta*, in order to move from one cell to another, if the target cell is directly visible or its location is known then all the neighboring cells are ignored, hence a speed-up is achieved. Phi* is further extension of Basic Theta* algorithm where it keeps knowledge of the predecessor and already visited cells by making use of two angles for each cell. Using the inherited property of Basic Theta*, the 
Phi* algorithm plans a path for the robot. The authors have used few other optimizations like Rectangular Symmetry Reduction and Jump Point Search for further improvements. These approaches are designed to achieve path planning for a single robot.

On the other hand, the authors in [27] proposed a path planning algorithm for multiple robots based on the mechanism of cooperation for accident free movement. The movement is made possible on the concept of "push" and "swap" model, where one agent pushes other to clear its shortest path to reach the destination. The shortest path is determined through traditional A* algorithm. On the other hand, when it becomes difficult for an agent to follow its shortest path towards the destination by pushing contemporary agent, then it swaps its position with the other agent to continue following the path.

Summary: We observe that heuristics and AI based approaches are well suited for robots with limited mobility requirements and may not work for visually impaired people. Most of these approaches require more time and computing resources to determine a suitable path except few. A* algorithm is simple and efficient, which makes it favorable to our problem. The majority evolutionary techniques are best in finding optimal path (global optimum solution) but at the expense of efficiency. However, Cuckoo search with Bat algorithm performs better in terms of execution time compared with its counterparts.

\subsection{Path Selection for Visually Impaired People}

A visually impaired person can travel from a source (or current place) to a destination by following a specific path. It is non-trivial to determine a customized or impairment aware path considering various specific mobility constraints, e.g. traffic flow, obstacles, sidewalks, curbs, and zebra crossings. The availability of limited information related to aforementioned constraints is one of the bottlenecks to develop an effective solution. In the following paragraphs, we study various approaches of choosing a suitable path for visually impaired people.

\subsubsection{Static Route Selection}

S. Kammoun et. al, introduced a route selection algorithm [29], it is among vary initial works in this domain and specifically designed for blind pedestrians, which uses annotated GIS database with four kinds of pre-classified objects namely points of interest, landmarks, walking areas, and visual points. In this approach, the authors construct a graph using source, destination, and intersections of the walking areas. The cost of each section (or edge in the graph) is defined based on user \& system preferences (presence of landmarks, point of interest, and visual points) and selection criteria (sidewalk presence and width, pedestrian crossing, and length of a section). This algorithm selects optimal path by solving minimization problem using well known shortest path strategy, i.e. Dijkstra Algorithm. The absence of availability of Geolocalized data is one of the bottlenecks to adopt this strategy, and it is non-trivial to annotate the dataset with pre-classified objects.

In another study [30][31], the authors utilized the concept of map transformation to generate customized maps, i.e. individualized route graphs, using OpenStreetMap (OSM) dataset. OSM is a crowdsourced project which is freely available for anyone to use. In this approach, the underlying navigation data is modified instead of tailoring the algorithms for specific groups of users. It means the navigation algorithms are generic while relying on the transformation for individualization. The OSM data format contains three basic entities namely nodes, ways and relations, where nodes and ways are quite different than normal graph nodes and edges. Each entity can have an arbitrary number of tags with key-value pairs. The authors introduced a layer on-top of the OSM data by representing streets with polygons instead of lines through 
predefined set of rules [31]. Further, they construct the route graph from OSM data including sidewalks and pedestrian crossings using set of transformation rules, refer to [30] for more details. The authors have used $\mathrm{A}^{*}$ algorithm to calculate the best routing path and general idea on guiding instructions is also discussed in terms of text messages, which can be static, dynamic or hybrid. This map transformation approach can be helpful in terms of easily identifying the walking area and pedestrian crossings, which was difficult through base OSM data representing streets as lines. However, we can not overlook the possibility of the absence of tagged information that eventually results in unavailability of such transformed OSM data.

Many researchers have developed effective strategies to determine optimal routes under certain constraints and scenarios. For instance, in [32], the authors utilized particle swarm optimization (PSO) strategy to find the best route which is as per their claim suitable for visually impaired people, however, we partially agree with that claim because visual impairment related aspects were not considered while finding the best route. One of the un-realistic assumptions in this study was that there are no obstacles on the ground, and the authors also assumed that waypoints along the pedestrian walkways are available in advance, which makes this strategy less attractive.

Summary: In this section, we reviewed prominent existing studies related to static route selection for a VIP. Despite the usefulness of those techniques, there exist various issues when it comes to find a best path. For instance, in [29] apart from the issue for non-availability of geolocalized data, the shortest path-based approach may overlook multi-criteria requirements while giving priority to select a path that is shortest in length. A shortest path can be longest as well, however, the computational complexity then increases while computing all possible shortest paths. Similarly, using OSM data [30][31] has its own advantages and disadvanteges. For instance, the authenticity of the tagged information provided by crowd. On the other hand, OSM is a freely available resource so is handy to use.

\subsubsection{Route Planning with Dynamics}

In [33] the authors present a system for trip planning that considers future traffic hazards. Incorporating the future traffic hazards into the path planning of VIPs is useful to determine the future trends on the selected path. A path for VIP may happen to be an ideal path at the moment, however, it may become crowded in the near future. Therefore, the system should suggest a path which remains optimal/valid for longer periods of time because a VIP requires sometime in order to get familiar with the path to be frequently used.

There are also some situations when travel of a VIP to a certain destination is initiated at different timings and also sensitive to the changes in the road conditions. A trip initiated on a certain path, for instance at 8 AM may have overcrowded traffic situations due to school and office timings. However, the same path can be easy to go for VIP at 10 AM. This very problem of path selection in smart cities is handled by authors in [34]. They have proposed a system called PreGo for real-time personalized routing in smart cities. Similarly, the authors in [35] also target a similar problem of dynamic and time-dependent route planning which takes into account the prediction of traffic status using historical data and live traffic status into account.

A greedy path planning algorithm [36] is developed for visually impaired people to smoothly navigate in a shopping mall by minimizing the number of turns and total distance. The idea can also be generalized to any other similar venue whose navigation map exists. The uniqueness of their proposal is that the algorithm finds the paths having a minimum number of turns. This very focus of the algorithm is useful since VIPs cannot move freely and they want a hassle-free straight path to reach their destination. In order to make a move, the algorithm computes an optimal path from the current location to the desired one. In the course of action, if an obstacle 
is detected, the algorithm re-routes the VIP by suggesting a new path, which is not easy. There is another limitation of the algorithm that it requires a list of obstacles as input, whereas the input list can have limited information and new obstacles can exist during movement. Nevertheless, the idea to have a minimum number of turns is practical.

Table 2. This table summarizes various AI-based and heuristic approaches in terms of type of path under consideration, computation strategy, information utilized, and application domain.

\begin{tabular}{|c|c|c|c|c|}
\hline Articles & $\begin{array}{l}\text { Type of } \\
\text { Path }\end{array}$ & $\begin{array}{l}\text { Computation } \\
\text { strategy }\end{array}$ & $\begin{array}{l}\text { Necessary information } \\
\text { used for Algorithm } \\
\text { Designing and } \\
\text { Execution }\end{array}$ & $\begin{array}{l}\text { Application } \\
\text { Domain }\end{array}$ \\
\hline $\begin{array}{l}\text { Shortest paths through a } \\
\text { reinforced random walk } \\
\text { [22] }\end{array}$ & $\begin{array}{l}\text { Shortest } \\
\text { Path }\end{array}$ & $\begin{array}{l}\text { Reinforced } \\
\text { Random Walk }\end{array}$ & $\begin{array}{l}\text { Model based on } \\
\text { Exponential and Poisson } \\
\text { distribution }\end{array}$ & $\begin{array}{l}\text { Electronic } \\
\text { Circuits } \\
\text { Networks }\end{array}$ \\
\hline $\begin{array}{l}\text { Neighbor-favoring weight } \\
\text { reinforcement to improve } \\
\text { random walk-based disease } \\
\text { gene prioritization [24] }\end{array}$ & $\begin{array}{l}\text { Frequently } \\
\text { visited } \\
\text { paths }\end{array}$ & $\begin{array}{l}\text { Modularity } \\
\text { Principle using } \\
\text { Random Walk } \\
\text { with Restart } \\
\end{array}$ & $\begin{array}{l}\text { Reinforcement of } \\
\text { Weights of the } \\
\text { Interactions or Edges }\end{array}$ & $\begin{array}{l}\text { Gene/Protein } \\
\text { Interaction } \\
\text { Network }\end{array}$ \\
\hline $\begin{array}{l}\text { Ant foraging and minimal } \\
\text { paths in simple graphs [23] }\end{array}$ & $\begin{array}{l}\text { Shortest } \\
\text { Path }\end{array}$ & $\begin{array}{l}\text { Reinforced } \\
\text { Random Walk }\end{array}$ & $\begin{array}{l}\text { Pheromone-induced } \\
\text { reinforcement along with } \\
\text { directional constraint }\end{array}$ & $\begin{array}{l}\text { General } \\
\text { Networks }\end{array}$ \\
\hline $\begin{array}{l}\text { Path planning with } \\
\text { modified } A^{*} \text { algorithm for a } \\
\text { mobile robot [26] }\end{array}$ & $\begin{array}{l}\text { Shortest } \\
\text { Path }\end{array}$ & A* Algorithm & $\begin{array}{l}\text { Grid representation of } \\
\text { space }\end{array}$ & $\begin{array}{l}\text { Path planning } \\
\text { algorithm for a } \\
\text { mobile robot }\end{array}$ \\
\hline $\begin{array}{l}\text { Push and Swap: Fast } \\
\text { Cooperative Pathfinding } \\
\text { with Completeness } \\
\text { Guarantees [27] }\end{array}$ & $\begin{array}{l}\text { Shortest } \\
\text { Path }\end{array}$ & $\begin{array}{l}\text { Any } \\
\text { state-of-the-art } \\
\text { Shortest Path } \\
\text { Algorithm }\end{array}$ & $\begin{array}{l}\text { Position of the other } \\
\text { agent }\end{array}$ & $\begin{array}{l}\text { Path planning } \\
\text { algorithm for } \\
\text { group of robots }\end{array}$ \\
\hline $\begin{array}{l}\text { Application of Dijkstra } \\
\text { algorithm in robot } \\
\text { path-planning [28] }\end{array}$ & $\begin{array}{l}\text { Shortest } \\
\text { Path }\end{array}$ & $\begin{array}{l}\text { Dijkstra } \\
\text { algorithm }\end{array}$ & $\begin{array}{l}\text { Number of dimensions of } \\
\text { the navigation space }\end{array}$ & $\begin{array}{l}\text { Path planning } \\
\text { algorithm for a } \\
\text { robot }\end{array}$ \\
\hline $\begin{array}{l}\text { Optimal Path Planning of } \\
\text { Mobile Robot Using Hybrid } \\
\text { Cuckoo Search-Bat } \\
\text { Algorithm [25] }\end{array}$ & $\begin{array}{l}\text { Obstacle } \\
\text { free } \\
\text { optimal } \\
\text { path }\end{array}$ & $\begin{array}{l}\text { Hybrid cuckoo } \\
\text { search and the } \\
\text { bat algorithm }\end{array}$ & $\begin{array}{l}\text { Local optimum solution } \\
\text { of one algorithm as input } \\
\text { for finding global } \\
\text { optimum solution }\end{array}$ & $\begin{array}{l}\text { Path planning } \\
\text { algorithm for a } \\
\text { robot }\end{array}$ \\
\hline
\end{tabular}

Interestingly, blind people and mobile robots share some aspects when it comes to finding a suitable route for navigation. However, we understand that the level of intelligence and objectives of navigation are quite different. Besides the differences, we are still interested to exhaust the possibilities of utilizing those techniques for navigation of living intelligent beings. Recent surveys [37][38] on path planning for robots provides a comprehensive view of those strategies. It is obvious that robots require more effort and information for navigation due to the absence of intelligence. The path planning process is classified into global or local and it consists of environment modelling, path searching, and path optimization. It is however, noted that the environment modelling aspect is quite different for robots compared to humans, as humans avail assistance from other senses.

Summary: Unlike previous section, we provide a review of existing studies for dynamic route selection that involves movement time, crowd status, weather condition, environment friendliness, and hazards that are going to happen in near future [33][34][35]. It is useful to note that sometimes crowd status also serves as a factor of environment friendliness, e.g. kids' noise on the route at certain time utilizes the concept of reinforced random walk in such a way that VIP is moving on a correct path. Considering path dynamics in such approaches is beneficial, however, they require more computational resources and internet connectivity, resulting in development of more power-hungry devises to be used during path navigation. Similarly, the idea to have minimum number of turns is practical, however, it can result in 
generating longer paths [36]. Finally, mimicking the scenario of robots with VIPs [37][38] cannot be mapped entirely in its spirit to that of VIPs, since VIPs as human are intelligent to feel the environment to make adjustments during their navigation.

\subsubsection{Heuristics and Al Techniques for Path Searching}

Path searching is one of the classic problems and researchers either adopt heuristics (Dijkstra, A*, D*, etc.) or artificial intelligence algorithms (Artificial Neural Network, Genetic Algorithms, Ant Colony Optimization, Particle Swarm Optimization, Reinforcement Learning, etc.) for global path planning. In local path planning, the path searching algorithms are different and it includes various approaches such as artificial potential field, behavior decomposition, cased-based learning, and rolling window.

A visually impaired person is mostly hesitant while making independent outdoor movements. This situation is especially notable in a less familiar environment. In this situation, the movement plan can benefit from the concept of reinforced random walk towards a successful journey. Random walk tells the probability of reaching a given destination from given source. Attaching the concept of reinforced learning to random walk means that a VIP makes use of prior knowledge/domain knowledge during the walk to improve the probability of reaching the destination [22] [24]. For instance, someone may have told a VIP that there is a school on the way towards the mosque from home. Hence noise of kids can be used as a reinforcement factor. So as soon as the VIP leaves home, he/she listens to kid's noise to plan her route, or the system recommends him/her a path having school on it. Whereas, such hints may help in planning a successful trip with shorter path length. In case of a VIP's independent movement, the concept of reinforcement, i.e. following the same trail, can be accomplished by utilization of domain knowledge or hints settings (aforementioned kids noise example or similar).

Summary: The reinforced random walk model [22][24] is useful because it is a feedback and reward-based approach. Such feedback always helps initially at the time of path computation and then assessing the quality of the chosen path in next usage. Since, it is a probabilistic model so prone to errors.

\subsubsection{Multicriteria Route Selection}

Sometimes, a VIP may need to visit multiple venues before reaching a destination. For instance, he may need to buy fruits, have a cup of tea from a coffee shop, offering prayers in the mosque, before reaching her friend's house. Whereas, many route planning engines allow individual searches for each action item and provide optimal however independent routes. Movement on these independent routes may be time consuming in terms of navigating to and froth between the desired places. Moreover, it is also possible that the recommended venues to satisfy the needs (quality fruit, nice hot tea etc) are not satisfactory. Therefore, the system must consider suggesting a route which is satisfactory in given criteria. A similar route selection problem is aimed in [39] where a smartphone application is designed which takes into account the user's satisfaction level and real time status of the traffic. Similarly, Traffic-aware route search (TARS) has been proposed in [40] which takes into account the points of interest/search terms along with variation in travel speed due to the effect of traffic conditions. In a similar study [41][42], the authors tackle the issues which arise in real time traffic aware systems when some undesirable routes are proposed e.g. passing through parking lots, residential areas and so on. The application of such undesirable detour for VIP may include passing over a bridge or crossing the highway and so on.

Taking a way forward from Big Data support, the authors in a study [43] propose specialized routes for VIPs by making use of OSM data. OSM provides details like longitude, latitude, list 
of paths, metadata about the objects like type of building. In this way, the data can be transformed into a directed graph having rich information of the ground realities. Using OSM and existing routing algorithms, the authors proposed approach suggest the paths for the VIPs which satisfy pre-defined restrictions like avoid passing a busy street.

The commute needs of physically disabled persons are also similar to VIPs in a sense that they cannot move freely, though, they can see any obstacle. To plan routes for physically disabled persons, the authors in [44] claim that the existing route planning algorithms are smart, however, they do not consider the profile of the users, physical disability in this case, at the time of path planning. The authors utilize ontologies of user and context along with the profile of the users as input to generate the recommendations.

Summary: In this section, we have discussed some of the studies considering multi-criteria requirements of a VIP. We understand that such multi-criteria aspects, discussed in [39][40][41], are practical because humans always have certain requirements to fulfill. Therefore, these approaches are crucial to develop a robust path selection approach for VIPs. The problem being discussed falls under the category of combinatorial optimization problems for which heuristics are designed and become computationally in-efficient at a certain point.

\section{Path Navigation Techniques}

Path navigation is the process of following a set of directional information step by step to reach the destination from starting a place. Path selection is the pre-requisite for path navigation, where path is either computed completely or partially depending on the underlying strategy. In this section, we discuss the navigation aspect of a user with pre-computed path information. We understand that the nature of mobility for a human is different than a robot due to inherent non-visual human senses. We study various approaches proposed in literature to guide an object (person/robot) for navigation from current place to a target location. Let we formally present the problem of path navigation in Definition 2, in accordance with Definition 1, so that we could review the existing studies accordingly.

Definition 2: Path Navigation Given a selected path pi, our aim is to develop a smooth navigation $\mathrm{n}$ to follow segments of the path $\mathrm{p}_{\mathrm{i}}$, where $\mathrm{n}$ satisfies $\mathrm{m}$ local constraints i.e. $\mathrm{m}=$ \{const $_{0}$, const $_{1}, \ldots$ const $_{\mathrm{i}}, \ldots$ const $_{\mathrm{k}}$ \}. The local constraints during navigation may include no collision while following the segment, least number of obstacles, and context-awareness.

\subsection{General Path Navigation}

There are various approaches developed and used for the mobility of the general public, however, we discuss selected ideas in the following passages. In [45], the authors have investigated the natural process of navigation using path integration among insects like ants. The core idea behind path integration is that the ant accumulates its distance from the nest upon movement, which they call vector navigation. However, in an unfamiliar terrain, the vector navigation approach is not applicable rather an alternative strategy called landmark navigation is preferred. Vector navigation and landmark navigation both can complement each other for effective navigation over long distances.

An evolutionary algorithm-based path planner is introduced in [46] to autonomously navigate unmanned aerial vehicle (UAV) in known and unknown environment. It calculates a curved path with desired properties in a 3-D rough terrain environment. The authors used genetic algorithm where chromosome genes represent coordinates of the controlling points. The path planner is either offline or online depending on whether the environment is known or 
unknown. The offline planner produces a predetermined path on which the UAV moves as defined by a B-Spline curve. In unknown environment, the online planner produces various smaller B-Spline curves connected smoothly and relies on the radar readings during its flight.

Robots or an avatar movement in a complex 3-D environment is studied in [47] through interactive navigation. The author precomputes a global roadmap of the environment, which is further analyzed to automatically produce a collision-free constrained path between source and destination locations. The proposed algorithm requires only the scene geometry and avatar orientation along with size parameters. The walkable points are generated on the global roadmap to construct a graph. This graph is used later to determine collision free path from one location to another. Once the path is determined then the avatar is propelled on this path. A runtime checker is enabled to continuously determine next points to be reachable from the current position of the avatar towards smooth transition. The navigation concept is native to vehicles (similar to robots) also and therefore an effective strategy, introduced in [48] to reduce the travel time of the vehicles in peak hours or during road congestion.

Summary: In the context of a blind person, offline planner approach [46] is suitable to construct an initial high-level navigation plan, i.e which trajectory be followed from source to destination. The offline planner mode cannot be used in its spirit due to the occurance of obstacles during navigation. Similarly, the approach of pre-computation of the global roadmap of the enviroment [47] resembles to the offline planner mode. Whereas, online planner makes sense when the blind person already started the journey and can follow smaller connecting B-Spline curves.

\subsection{Path Navigation for Visually Impaired People}

Path navigation for visually impaired people is nontrivial as it requires to guide the user step by step with an understanding of the surrounding by following a particular path. It becomes more complex when blind person experiences a deviation in the selected path while moving. In order to overcome the issue of localization, researchers have adopted various technologies and developed techniques for indoor and outdoor mobility such as GPS services, low-energy location aware beacons, map construction for locality, etc. In the following paragraphs, we discuss some of the representative ideas briefly.

It is one of the viable strategies, especially in indoor environments, to establish a network of low-energy beacons for guidance. The authors in [49] make use of beacons for guidance of VIPs. They propose an information system, named as BlindDroid, based on the location information. BlindDroid is also accompanied with question answering module, and details of the surroundings. The information of the surroundings consists of the metadata information of each location to facilitate the movements. The system performs outdoor localization using GPS and indoor localization through beacons.

The understanding of the surrounding environment, either indoor or outdoor, is one of the essential tasks in any mobility service or application for VIPs. It begins with the scene capturing process normally done by artificial vision, e.g. stereo camera, module followed by object detection component from the captured scene. Once the understanding is established then system suggests or guide the person with appropriate navigation instructions, e.g. walk 5 meter straight or turn left. The processing and interpretation of the visual scene is nontrivial and requires specialized techniques and algorithms to achieve acceptable results. One of the useful tools for visual scene analysis is SpikeNet [50], which allows to process video stream in real-time to locate visual features. However, scene understanding may not suffice for a blind person to navigate. Therefore, the authors in another study [51] integrated the artificial vision with GPS and modified Geographical Information System (GIS) [52]. 
Similarly, the authors in [53] propose an intelligent walking system for VIPs in a university environment. Their proposed system makes use of communication between mobile devices held by a VIP, Central Navigation System that is a server PC to store maps and navigation routes of the premises, and beacons that are small and low-cost hardware components to frequently transmit signals among other devices for mid-way location estimation.

Summary: The ideas of beacons placement for navigation [49] and mantaining maps is quite expensive and not realistic for outdoor navigation due to complex and dynamic environment. However, it needs to be integrated with the concept of offline planner mode of [46] or pre-computation of the global roadmap of the enviroment [47] so that an end to end tarjectory is pre-computed and then this live streaming mode [50] is used for collision free and safe movement. The integration of GPS [51] and GIS [52] increases the accuracy and performance of these pre-compuation approaches.

\subsection{Context-aware Mobility}

The context plays a crucial role in determining effective navigation path for visually impaired people [54], therefore, we study in depth the strategies developed in the past for context awareness. Some of the relevant studies are briefly described in the following paragraphs.

A new model for human trajectory prediction is presented in [55] which is able to take advantage of both human-human and human-space interactions. The future trajectory of humans is generated by observing their past positions and interactions with the surroundings. To this end, the author proposed a "context-aware" LSTM model, which can learn and predict human motion in crowded spaces such as a sidewalk, a museum or a shopping mall. Context is defined using static elements (human-static objects) of the scene and dynamics agents (human-human interactions) in the same scenario. The proposed model observes the past positions of a human and interactions with the surroundings to predict near future trajectory.

A context-aware system considers a user's profile and his current contextual condition for recommendation [56]. An extension to this work took advantage of the additional information that mobile devices provide, mainly their ubiquitous nature and the implicit knowledge of the user's physical location obtained by its GPS (Global Positioning System). However, additional contextual information such as direction, trajectory, speed, etc., was not considered, in spite of the fact that they are very interesting context features for tourism purposes.

Context based on mobile phones can be divided into three parts, as follows as per the study in [57], namely user environment (location, preference, experiment, social relations, and so forth), mobile environment (device suitable for users to input or display, network, Bluetooth, and so forth), and physical environment (weather, date, noisy, etc.). In [58], the authors presented a context-aware adaptive interface for mobile location-based systems. In [59], the proposed method identifies a driver's contexts and then identifies driving preferences for each context using historical trajectories from the driver. Micro-navigation systems [60] detect the objects that can be a barrier for the blind and visually impaired (BVI) people. In the same article, the authors have introduced a macro-navigation system to assist BVI by localizing them and providing turn-by-turn navigation instructions.

Summary: The approach used in [55] observes the past positions of a human and interactions with the surroundings to predict near future trajectory. However, making use of such prediction during the movement is not effective due to occurrence of different types of obstacles with varying positions in the surroundings, which is not the case with indoor environment. The context identification through mobile phone data [57] and GPS information [56] is also difficult to adopt in indoor settings. 


\section{Applications and Systems for Guidance}

In this section, we describe related applications and systems used or assist in the navigation of the blind people. Recent surveys [13] [12] [14] [61] discussed various kinds of systems and applications developed for VIP. These systems and applications are either the outcomes of research work in this domain as prototypes or entirely as business-oriented products. It may also include free and open source systems. The discussion in this section highlights key aspects these systems cover in terms of guiding VIP and also provide insights to improve them for better guidance. We briefly discuss some of the related and already developed applications, systems, and devices as highlighted in [62]. These solutions are categorized into general purpose, object recognition based, navigation related, and specialized systems and devices.

\subsection{General Object Recognition based Applications}

Be My Eyes [63] is a useful application for partially sighted or blind people where a VIP is helped remotely by a sighted volunteer or company representative. It requires volunteers and VIPs to configure and install this application on their smartphones. However, there are a few drawbacks, which limits this application to be acceptable by a wide range of users, such as the availability of the volunteer all the time, internet connectivity that is not strong and reliable in all regions of commute, and chance of unavailability of volunteer with required language.

TapTapSee [64] can be used at the time of travelling of VIP to detect the object on the way. This application uses a camera to take a snapshot of the object and conveys its understanding to the user through voice-over function. The user needs to tap the screen to capture the scene/object with any arbitrary angle. Afterwards, the system analyzes the captured scene, using Cloud Sight Image Processing Library [65], to identify the objects in seconds. The resultant identification is communicated through audible voice to the impaired user. This application captures one scene at a time with sequential numbering. The captured image is transmitted to the dedicated server and results are communicated back through the application. This whole process takes a couple of seconds and therefore not suitable for navigation purpose, which requires real-time response. The voice-over function speak-out the result of the last processed image even if there are multiple scenes analyzed prior to it. The application is not mature enough to recognize the semantics of the scene accurately. In our understanding, it may work in an indoor environment effectively to detect various objects but not a good choice for outdoor scenarios. The detailed analysis on TapTapSee and other is presented in [66].

iDentifi [67] is also similar to TapTapSee which can be used during outdoor movement for shopping like situations. It uses the camera of the cell phone to recognize almost any object, the color, text, facial expressions and then delivers an audible message to a blind user. SeeingAI [68] also provides almost similar functionalities to that of TapTapSee. However, both of these applications analyze individual images with a high response time, i.e. between 2-10 seconds depending on the speed of internet connectivity, which makes them not suitable for path navigation. There are other applications, such as BeSpecular [69], which help the visually impaired people by enabling them to take a snapshot of the scene and share it through application with the community of sighted volunteers. The available volunteer in the community listens to the audio message along with the visual scene and responds with text or voice message, which is sent back to the originating impaired person. The bottleneck remains the same of volunteer's availability and response time. 


\subsection{Navigation Oriented Applications}

In this section, we discuss various applications designed for navigation purposes. Microsoft soundscape [70] is one of the potential applications for guiding visually impaired people through sound. This application provides an interaction between visually impaired people and the surroundings to enrich ambient awareness. It utilizes spatial audio to call out the landmarks in relation to the navigating person. The navigation information is collected through the crowdsourced data, i.e. OpenStreetMap. It also requires the user to set the audio beacons for localization, which is one of the bottlenecks towards ease of use. It does not consider the real-time situation or moving objects around blind person.

NavCog3 [71][72] is a smartphone-based indoor navigation assistant for visually impaired people. This system aims to provide an assistive mechanism to visually impaired for smooth movement in a large environment like a grand shopping mall, etc. NavCog3 receives input from the target users, using speech-to-text method and provides nearby points of interest (POI) information. As soon as POI are set, point-to-point movement is enabled by placing Bluetooth low-energy (BLE) beacons with a distance of 7-10 meters. A very similar idea for autonomous indoor navigation is presented in WayFindr system [73] [74]. The concept of beacons is also used to assist the VIPs to identify the possible passage and then navigate accordingly. WayFindr establishes standard guidelines to help the practitioners and developers in this domain.

Intersection Explorer [75][76] speaks the layout of the streets, crossing points and surroundings of the current location of a VIP. It allows users to not only preview their journey in a street view style but enable partially sighted people to rehearse paths with ease and comfort. It provides an easy to use touch interface for visually impaired people to determine the road or path intersections and street layouts. This application usually malfunctions while navigating, i.e. force to close, and incurs unacceptable error in location accuracy.

A slightly more enhanced version is BlindSquare [77] which speaks out the neighborhood, points of interest in the surroundings, and street crossings as well. It is a widely used and proprietary GPS based application. This application utilizes the navigation information from third party applications, such as OpenStreetMaps and FourSquare to acquire surrounding information, along with the specially designed algorithms to manipulate the available data.

Arianna Navigation [78] is another alternative application which facilitates both indoor and outdoor navigation through a dedicated simple infrastructure composed of colored paths painted on the floor. Navigation in various indoor places like hospitals, malls, airports and outdoor places is facilitated by vibrational signals to correct the direction. Lazarillo [79] is GPS enabled mobile applications to guide blind users through voice messages. It uses international databases of maps, therefore works worldwide, and GPS service to retrieve current location and information regarding cafes, bus stations, banks, restaurants and more. It provides directions to desired destinations using aforementioned information. One of the key issues we notice, while using the application, is that the availability of landmarks information is limited to well established regions of the world. Moreover, this application does not consider dynamically moving objects in the surroundings. Lazzus [80] is no different which entirely relies on third party navigational applications such as Google Maps, Bing Maps etc.

The idea of NaviLens [81] application for VIPs is unique in a sense that they do not rely on the information about surroundings provided by the existing infrastructure and services, rather, they create their own. The authors transform the navigation information in the form of unique bi-dimensional (or BIDI) codes. These codes are placed as per the encoded directional information in the surroundings. During the journey of a VIP, the user moves around the 
smartphone's camera to find and capture the available code(s) nearby to hear the related information. The augmentation of the environment becomes the bottleneck to this approach.

Table 3. Comparison of various path guidance applications and systems in terms of their capabilities and the quality of supported functionality.

\begin{tabular}{lcccccccc} 
Apps \& Systems & GPS & $\begin{array}{l}\text { Audio/ } \\
\text { Heptic }\end{array}$ & Image & Video & $\begin{array}{l}\text { Object } \\
\text { Detection }\end{array}$ & Free & Autonomy & Usability \\
\hline NavCog3 [72] & No & Yes & No & No & No & Yes & Yes & MED \\
WayFindr [74] & No & Yes & No & No & No & Yes & Yes & MED \\
BeMyEyes [63] & No & Yes & Yes & Yes & No & Yes & No & MED \\
TapTapSee [64] & No & Yes & Yes & No & Yes & Yes & Yes & LOW \\
iDentifi [67] & No & Yes & Yes & No & Yes & Yes & Yes & LOW \\
SeeingAI [68] & No & Yes & Yes & Yes & Yes & Yes & Yes & MED \\
BeSpecular [69] & No & Yes & Yes & No & No & Yes & No & LOW \\
Soundscape [70] & Yes & Yes & No & No & No & Yes & Yes & LOW \\
BlindSquare [77] & Yes & Yes & No & No & No & No & Yes & MED \\
Lazarillo [79] & Yes & Yes & No & No & No & Yes & Yes & LOW \\
Intersection & Yes & Yes & No & No & No & Yes & Yes & MED \\
Explorer [75] & & & & & & & & \\
Lazzus [80] & Yes & No & No & No & No & Yes & No & LOW \\
Arianna & No & Yes & No & Yes & Yes & Yes & No & LOW \\
Navigatio [78] & & & & & & & & \\
NaviLens [81] & No & Yes & Yes & No & Yes & Yes & Yes & MED \\
Sunu [82] & No & Yes & No & No & Yes & No & Yes & LOW \\
OrCam [83] & No & Yes & Yes & No & Yes & No & No & LOW \\
Blaid [84] & Yes & Yes & Yes & No & Yes & No & Yes & MED \\
eSight [85] & No & Yes & Yes & No & Yes & No & Yes & MED \\
iBionics [86] & No & Yes & Yes & No & Yes & No & Yes & MED \\
\hline
\end{tabular}

We provide an overview of comparative analysis for all the applications and systems, discussed in this section, with respect to predefined criteria in Table 3 . The criterion is selected based on suitability of these applications and systems for visually impaired people towards autonomous navigation, which is the main focus of this article. GPS criterion depicts whether the application or system supports location-aware services or not. These applications interact with the users through either audible instructions or haptic feedback. Not all applications and systems analyze images and video data to observe the surroundings and develop an understanding for the VIPs. The understanding of the surroundings depends upon effectively detecting and identifying the objects. Object detection is a key step towards scene understanding, where the majority of the techniques under consideration detect various kinds of objects from individual images. However, few approaches detect objects continuously from a series of images taken through built-in camera. Most of the applications are available free of cost on either one or both platforms, i.e. iOS and Android, except BlindSquare [77]. It is critical for any application or system to be autonomous so that a visually impaired person feels more independent. This is one of the major factors to influence the usability of any system or application, as depicted in the last column of the comparison table. It is interesting to know that existing solutions are either more focused on non-visual information, e.g. GPS signals and navigational semantics, or visual content to understand the surroundings. In our understanding, 
visual content analysis is a complex task compared with the analysis of non-visual information towards navigational guidance, therefore, many aspects in visual content analysis are still under consideration.

\subsection{Specialized Devices}

We enumerate related ideas and products as wearable devices in the following discussion, readers may refer to a detailed survey on wearable assistive devices for VIPs [13]. Sunu [82] is a wearable wrist device which helps a visually impaired or with low vision about obstacles in the surroundings. It utilizes sonar technology, or echolocation, to detect obstacles nearby up to 16 feet or 5.5 meters away with acceptable precision. The haptic feedback helps the user to understand how far the object is. This device is not a replacement for white-cane or dog but complements them. It supports haptic compass and GPS enabled application through smartphone to explore unfamiliar regions. Given all these capabilities, this device overlooks the diversity and nature of obstacles and path guidance properties for visually impaired such as street or road intersections, curbs, and road-crossings to name a few.

OrCam [83] is a wearable AI vision device equipped with a miniature camera and a speaker. It can be easily mounted to eyeglass frames. It involves capturing images of interest through built-in camera and speak out the recognized optical characters. Though, this device is not capable of helping visually impaired people is their commute, however, it can assist the blind person to understand the various cues along with the localization services, as also suggested in [87]. Blaid project [84] by Toyota is another expected wearable product for blind and VIPs to overcome their mobility challenges. The company promise to equip the shoulder wearable device with camera to capture and understand the surroundings along with the communication components like speakers, microphone, vibration motors. However, the product yet to be realized and no detailed information about path computation and navigation technique is available.

There are few initiatives to restore the seeing capability of a blind person through technology advancements. eSight [85] is one of such products in the market, which interacts with the human body to generate required information after processing high definition captured scene. This device looks like normal glasses and can be used by any low-vision or blind person. iBionics [86] belongs to the same category of eSight where it works like Retina of the human eye. Though the human eye cannot see but the brain does through this implanted device. The core features of this device include high acuity, superior diamond-based biomaterial, highly flexible simulator chip, and wireless technology. The retinal implant, which is enclosed in a diamond box to stimulate the retina, receives the image for processing and generates corresponding neural response to be received and understood by the brain. The underlying technology for these kinds of devices still lacks generality and is limited to selected scenarios, e.g. read text and detect faces. One of the key issues with these devices is that the poor people cannot afford to buy in our community.

\section{Open Issues and Research Directions}

In this section, we discuss various open issues related to our topic and potential research directions towards the development of an effective solution to provide an autonomous mobility for visually impaired people. We understand that the following issues are critical and need to be addressed in future due to lack of efforts in literature.

Safe and interesting path (SIP) discovery approach: The current developments in determining suitable paths for VIPs are not adequate. It is imperative to develop an effective 
approach to find safe and interesting path, i.e. with fewer obstacles/hurdles and meeting various user interests such as POIs, between source and destination for a given set of constraints specific to impaired persons. Defining the representative criterion for VIPs is itself an issue that needs attention. The unavailability of information related to VIP constraints in contemporary geographic information systems (GIS) is an obstacle for SIP discovery, therefore, a systematic solution is necessary to capture those static and dynamic travel constraints.

Sharing travel experience through SIPs repository and prediction algorithm: We observe that there is lack of effort in maintaining the travel experience of VIPs, which has the potential to become a useful resource for others. We need to develop such repository of SIPs by capturing VIPs movements through handheld devices. The dynamicity of surroundings may result in repeated deviations to the initially estimated SIP and determining new SIP is inevitable. A prediction algorithm based on the SIP repository can improve performance of new SIP estimations and seems to be another direction to follow.

Realtime hazard detection and object identification on smart devices: The complexity of the task and available computation resources make it difficult to perform static and dynamic object detection and identification in real-time. Few initiatives exist in literature to provide limited functionality in this context. A robust detection and identification mechanism on smart phones are still open challenges for researchers and developers. We need an algorithm and model to detect various kinds of obstacle/hazards instantly to take respective measures by the impaired person while navigating in unknown territory. This algorithm expects to use vital information, i.e. visual and non-visual, to make decisions quickly, however, research community is not confident enough on integration of visual and non-visual content. What aspects needed to build such model and algorithm is yet to discover.

Context-aware navigation system: A few efforts have been made to acquire contextual information from interactions between humans and physical-spaces or features associated with the environmental entities. Researchers have used audio and haptic based tools to communicate the contextual understanding with the VIPs. However, capturing interaction information from humans and physical spaces on the go and develop an understanding for VIPs is still an on-going research. A realistic approach is required to determine the context of the surroundings (knowledge of surroundings like passing by superstore, footpath is narrow, crowded place etc.) through visual and non-visual data, which helps the users to navigate smoothly.

A comprehensive system with smart navigation: After analyzing various related applications and systems, there is no reasonable solution for smartphones to provide real-time outdoor mobility for VIPs with continuous hazard detection and identification in unknown environments. We noticed almost no usage of contextual information in those applications. Therefore, a system is required to provide a comprehensive solution for navigation problem in unknown territories. Smart devices equipped with essential tools and commodity sensors make it a best choice to realize such solution.

\section{Conclusion}

One of the key challenges for visually impaired people is to have safe and smooth commute. In order to assist the development of the specifically designed systems for blind people, we have performed a detailed survey on existing path planning algorithms and systems. Our survey is versatile in a sense that we have covered both the general approaches and VIP's movement specific ones for path planning, establishment, and suggestion. The inclusion of general 
approaches helps us consider the variety of use cases while designing assistive services for VIPs. In order to reduce gap between theoretical and practical solutions, we presented brief discussion on various existing applications and specialized devices tailored to capture the needs of blind people. Based on this survey, we understand that there is a significant gap towards the developed solutions and actual needs of visually impaired people in terms of richer and comfortable paths for safe and smooth navigation.

\section{References}

[1] Elmannai, W.M., Elleithy, K.M., "A highly accurate and reliable data fusion framework for guiding the visually impaired,” IEEE Access, 6, 33029-33054, 2018. Article (CrossRef Link)

[2] Bourne, R.R., Flaxman, S.R., Braithwaite, T., Cicinelli, M.V., Das, A., Jonas, J.B., Keeffe, J., Kempen, J.H., Leasher, J., Limburg, H., others, "Magnitude, temporal trends, and projections of the global prevalence of blindness and distance and near vision impairment: a systematic review and meta-analysis,” The Lancet Global Health, 5, e888-e897, 2017. Article (CrossRef Link)

[3] Mobasheri, A., Sun, Y., Loos, L., Ali, A.L., "Are crowdsourced datasets suitable for specialized routing services? Case study of OpenStreetMap for routing of people with limited mobility," Sustainability, 9, 997, 2017. Article (CrossRef Link)

[4] Froehlich, J.E., Brock, A.M., Caspi, A., Guerreiro, J., Hara, K., Kirkham, R., Schöning, J., Tannert, B., "Grand challenges in accessible maps," interactions, 26, 78-81, 2019. Article (CrossRef Link)

[5] Brock, A.M., Froehlich, J.E., Guerreiro, J., Tannert, B., Caspi, A., Schöning, J., Landau, S., "Sig: Making maps accessible and putting accessibility in maps," in Proc. of Extended Abstracts of the 2018 CHI Conference on Human Factors in Computing Systems, pp. 1-4, 2018.

Article (CrossRef Link)

[6] Hill, M.R., Spatial structure and decision-making aspects of pedestrian route selection through an urban environment, $\mathrm{PhD}$ thesis, University of Nebraska-Lincoln, 1982. Article (CrossRef Link)

[7] Hill, M.R., Walking, crossing streets and choosing pedestrian routes: A survey of recent insights from the social/behavioral sciences, PhD thesis, University of Nebraska-Lincoln, 1984.

Article (CrossRef Link)

[8] Otte, M.W., "A survey of machine learning approaches to robotic path-planning," Computer Science, 2009. Article (CrossRef Link)

[9] Garroppo, R.G., Giordano, S., Tavanti, L., "A survey on multi-constrained optimal path computation: Exact and approximate algorithms,” Computer Networks, 54, 3081-3107, 2010. Article (CrossRef Link)

[10] Kanimozhi, V., Karthik, S., "A survey on multi-constrained optimal path selection schemes and authentication schemes for VANET," in Proc. of 2017 11th International Conference on Intelligent Systems and Control (ISCO). IEEE, pp. 25-29, 2017. Article (CrossRef Link)

[11] Noreen, I., Khan, A., Habib, Z., "Optimal path planning using RRT* based approaches: a survey and future directions," Int. J. Adv. Comput. Sci. Appl, 7, 97-107, 2016. Article (CrossRef Link)

[12] Elmannai, W., Elleithy, K., "Sensor-based assistive devices for visually impaired people: Current status, challenges, and future directions," Sensors, 17, 565, 2017. Article (CrossRef Link)

[13] Tapu, R., Mocanu, B., Zaharia, T., "Wearable assistive devices for visually impaired: A state of the art survey," Pattern Recognition Letters, 2018. Article (CrossRef Link)

[14] Real, S., Araujo, A., "Navigation Systems for the Blind and Visually Impaired: Past Work, Challenges, and Open Problems," Sensors, 19, 3404, 2019. Article (CrossRef Link)

[15] Tiakas, E., Papadopoulos, A.N., Manolopoulos, Y., "Skyline queries: An introduction," in Proc. of 2015 6th International Conference on Information, Intelligence, Systems and Applications (IISA). IEEE, pp. 1-6, 2015. Article (CrossRef Link)

[16] Zheng, S., Zaman, A., Morimoto, Y., "Friend Recommendation by Using Skyline Query and Location Information," Bulletin of Networking, Computing, Systems, and Software, 5, 68-72, 2016. Article (CrossRef Link) 
[17] Tian, Y., Lee, K.C., Lee, W.C., "Finding skyline paths in road networks," in Proc. of the 17th ACM SIGSPATIAL International Conference on Advances in Geographic Information Systems, pp. 444-447, 2009. Article (CrossRef Link)

[18] Kriegel, H.P., Renz, M., Schubert, M., "Route skyline queries: A multi-preference path planning approach," in Proc. of 2010 IEEE 26th International Conference on Data Engineering (ICDE 2010). IEEE, pp. 261-272, 2010. Article (CrossRef Link)

[19] Kossmann, D., Ramsak, F., Rost, S., "Chapter 25 - Shooting Stars in the Sky: An Online Algorithm for Skyline: An Online Algorithm for Skyline Queries,” in Proc. of VLDB '02: Proceedings of the 28th International Conference on Very Large Databases; Bernstein, P.A., Ioannidis, Y.E., Ramakrishnan, R., Papadias, D., Eds., Morgan Kaufmann: San Francisco, pp. 275 - 286, 2002. Article (CrossRef Link)

[20] Papadias, D., Tao, Y., Fu, G., Seeger, B., “An optimal and progressive algorithm for skyline queries,” in Proc. of the 2003 ACM SIGMOD international conference on Management of data, pp. 467-478, 2003. Article (CrossRef Link)

[21] Bartolini, I., Ciaccia, P., Patella, M., "SaLSa: computing the skyline without scanning the whole sky," in Proc. of the 15th ACM international conference on Information and knowledge management, pp. 405-414, 2006. Article (CrossRef Link)

[22] Li, D., "Shortest paths through a reinforced random walk," 2011.

[23] Vela-Perez, M., Fontelos, M., Velazquez, J., “Ant foraging and minimal paths in simple graphs,” arXiv preprint arXiv:1103.1612, 2011. Article (CrossRef Link)

[24] Le, D.H., Kwon, Y.K., "Neighbor-favoring weight reinforcement to improve random walk-based disease gene prioritization," Computational biology and chemistry, 44, 1-8, 2013. Article (CrossRef Link)

[25] Saraswathi, M., Murali, G.B., Deepak, B., “Optimal Path Planning of Mobile Robot Using Hybrid Cuckoo Search-Bat Algorithm,” Procedia Computer Science, 133, 510 - 517, 2018. Article (CrossRef Link)

[26] DuchoE, F., Babineca, A., Kajana, M., BeE` oa, P., Floreka, M., Ficoa, T., Jurišicaa, L., "Path planning with modified a star algorithm for a mobile robot,” Procedia Engineering, 96, 59-69, 2014. Article (CrossRef Link)

[27] Luna, R.J., Bekris, K.E., "Push and swap: Fast cooperative path-finding with completeness guarantees," in Proc. of Twenty-Second International Joint Conference on Artificial Intelligence, pp. 294-300, 2011. Article (CrossRef Link)

[28] Wang, H., Yu, Y., Yuan, Q., “Application of Dijkstra algorithm in robot path-planning,” in Proc. of 2011 second international conference on mechanic automation and control engineering. IEEE, pp. 1067-1069, 2011. Article (CrossRef Link)

[29] Kammoun, S., Dramas, F., Oriolaand, B., Jouffrais, C., "Route selection algorithm for blind pedestrian,” in Proc. of ICCAS 2010. IEEE, pp. 2223-2228, 2010. Article (CrossRef Link)

[30] Schmitz, B., Ertl, T., "Individualized route planning and guidance based on map content transformations," in Proc. of International Conference on Computers for Handicapped Persons. Springer, pp. 120-127, 2014. Article (CrossRef Link)

[31] Schmitz, B., Ertl, T., "Creating task-specific maps with map content transformations. Proceedings of the 1st ACM SIGSPATIAL,” in Proc. of International Workshop on MapInteraction, pp. 84-90, 2013. Article (CrossRef Link)

[32] Yusof, T., Toha, S.F., Yusof, H.M., "Path planning for visually impaired people in an unfamiliar environment using particle swarm optimization,” Procedia Computer Science, 76, 80-86, 2015. Article (CrossRef Link)

[33] Liebig, T., Piatkowski, N., Bockermann, C., Morik, K., "Predictive Trip Planning-Smart Routing in Smart Cities,” in Proc. of EDBT/ICDT Workshops, pp. 331-338, 2014.

[34] Hendawi, A.M., Rustum, A., Ahmadain, A.A., Hazel, D., Teredesai, A., Oliver, D., Ali, M., Stankovic, J.A., "Smart personalized routing for smart cities," in Proc. of 2017 IEEE 33rd International Conference on Data Engineering (ICDE). IEEE, pp. 1295-1306, 2017.

Article (CrossRef Link) 
[35] 35. Baum, M., Dibbelt, J., Pajor, T., Wagner, D., "Dynamic time-dependent route planning in road networks with user preferences,” in Proc. of International Symposium on Experimental Algorithms. Springer, pp. 33-49, 2016. Article (CrossRef Link)

[36] Nandini, D., Seeja, K., “A novel path planning algorithm for visually impaired people,” Journal of King Saud University-Computer and Information Sciences, 31, 385-391, 2019. Article (CrossRef Link)

[37] Zhang, H., Lin, W., Chen, A., “Path Planning for the Mobile Robot: A Review,” Symmetry, 10, 450, 2018. Article (CrossRef Link)

[38] Patle, B., Pandey, A., Parhi, D., Jagadeesh, A., others., “A review: On path planning strategies for navigation of mobile robot,” Defence Technology, vol. 15, no. 4, pp. 582-606, 2019. Article (CrossRef Link)

[39] Levin, R., Kanza, Y., "Interactive traffic-aware route search on smartphones," in Proc. of the First ACM SIGSPATIAL International Workshop on Mobile Geographic Information Systems, pp. 1-8, 2012. Article (CrossRef Link)

[40] Levin, R., Kanza, Y., “TARS: traffic-aware route search,” GeoInformatica, 18, 461-500, 2014. Article (CrossRef Link)

[41] Delling, D., Schieferdecker, D., Sommer, C., "Traffic-Aware Routing in Road Networks," in Proc. of 2018 IEEE 34th International Conference on Data Engineering (ICDE). IEEE, pp. 1543-1548, 2018. Article (CrossRef Link)

[42] Xu, J., Guo, L., Ding, Z., Sun, X., Liu, C., "Traffic aware route planning in dynamic road networks," in Proc. of International Conference on Database Systems for Advanced Applications. Springer, pp. 576-591, 2012. Article (CrossRef Link)

[43] Koester, D., Awiszus, M., Stiefelhagen, R., "Mind the gap: Virtual shorelines for blind and partially sighted people,” in Proc. of the IEEE International Conference on Computer Vision Workshops, pp. 1443-1451, 2017. Article (CrossRef Link)

[44] Bravo, A.P., Giret, A., "Recommender System of Walking or Public Transportation Routes for Disabled Users," in Proc. of International Conference on Practical Applications of Agents and Multi-Agent Systems. Springer, pp. 392-403, 2018. Article (CrossRef Link)

[45] Collett, M., Collett, T.S., “How do insects use path integration for their navigation?,” Biological cybernetics, 83, 245-259, 2000. Article (CrossRef Link)

[46] 46. Nikolos, I.K., Valavanis, K.P., Tsourveloudis, N.C., Kostaras, A.N., "Evolutionary algorithm based offline/online path planner for UAV navigation,” IEEE Transactions on Systems, Man, and Cybernetics, Part B (Cybernetics), 33, 898-912, 2003. Article (CrossRef Link)

[47] Salomon, B., Garber, M., Lin, M.C., Manocha, D., "Interactive navigation in complex environments using path planning,” in Proc. of the 2003 symposium on Interactive 3D graphics, pp. 41-50, 2003. Article (CrossRef Link)

[48] Chen, W., Zhu, S., Li, D., "VAN: Vehicle-assisted shortest-time path navigation,” in Proc. of The 7th IEEE International Conference on Mobile Ad-hoc and Systems (IEEE MASS 2010). IEEE, pp. 442-451, 2010. Article (CrossRef Link)

[49] Cecílio, J., Duarte, K., Furtado, P., "BlindeDroid: An Information Tracking System for Real-time Guiding of Blind People,” Procedia Computer Science, vol. 52, pp. 113-120, 2015. Article (CrossRef Link)

[50] Thorpe, S.J., Guyonneau, R., Guilbaud, N., Allegraud, J.M., VanRullen, R., “SpikeNet: Real-time visual processing with one spike per neuron,” Neurocomputing, 58-60, 857-864, 2004. Article (CrossRef Link)

[51] Brilhault, A., Kammoun, S., Gutierrez, O., Truillet, P., Jouffrais, C., "Fusion of artificial vision and GPS to improve blind pedestrian positioning," in Proc. of 2011 4th IFIP International Conference on New Technologies, Mobility and Security. IEEE, pp. 1-5, 2011. Article (CrossRef Link)

[52] Loomis, J.M., Golledge, R.G., Klatzky, R.L., Speigle, J.M., Tietz, J., "Personal guidance system for the visually impaired," in Proc. of the first annual ACM conference on Assistive technologies, pp. 85-91, 1994. Article (CrossRef Link) 
[53] Kose, U., Vasant, P., "Better campus life for visually impaired University students: intelligent social walking system with beacon and assistive technologies,” Wireless Networks, pp. 1-15, 2018. Article (CrossRef Link)

[54] Gharani, P., Karimi, H.A., "Context-aware obstacle detection for navigation by visually impaired,” Image and Vision Computing, 64, 103-115, 2017. Article (CrossRef Link)

[55] Bartoli, F., Lisanti, G., Ballan, L., Del Bimbo, A., "Context-aware trajectory prediction,” in Proc. of 2018 24th International Conference on Pattern Recognition (ICPR). IEEE, pp. 1941-1946, 2018. Article (CrossRef Link)

[56] Barranco, M.J., Noguera, J.M., Castro, J., Martínez, L., “A context-aware mobile recommender system based on location and trajectory,” Management intelligent systems; Springer, pp. 153-162, 2012. Article (CrossRef Link)

[57] Gellersen, H.W., Handheld and Ubiquitous Computing," in Proc. of First International Symposium, HUC'99, Karlsruhe, Germany, September 27-29, 1999, Springer Science \& Business Media, 1999. Article (CrossRef Link)

[58] Feng, J., Liu, Y., "Intelligent context-aware and adaptive interface for mobile LBS," Computational intelligence and neuroscience, vol. 2015, p. 10, 2015. Article (CrossRef Link)

[59] Yang, B., Guo, C., Ma, Y., Jensen, C.S., “Toward personalized, context-aware routing,” The VLDB Journal, 24, 297-318, 2015. Article (CrossRef Link)

[60] Katz, B.F., Kammoun, S., Parseihian, G., Gutierrez, O., Brilhault, A., Auvray, M., Truillet, P., Denis, M., Thorpe, S., Jouffrais, C., "NAVIG: augmented reality guidance system for the visually impaired," Virtual Reality, 16, 253-269, 2012. Article (CrossRef Link)

[61] Darvishy, A., “Assistive technologies: short overview and trends," in Proc. of 1st Swiss conference on barrier-free communication, Winterthur, 15-16 September 2017. ZHAW Zürcher Hochschule für Angewandte Wissenschaften, pp. 52-55, 2018. Article (CrossRef Link)

[62] NoisyVision.org. Apps and devices for blind and visually impaired people. Web (CrossRef Link). Accessed: 2020-02-02.

[63] Wiberg, H.J. Be-My-Eyes: Bringing sight to blind and low-vision people. Web (CrossRef Link). Accessed: 2020-02-02.

[64] TapTapSee: Assistive Technology for the Blind and Visually Impaired. Web (CrossRef Link). Accessed: 2020-02-02.

[65] CloudSight: Image Processing Library. Web (CrossRef Link). Accessed: 2020-02-02.

[66] Holton, B., “A review of the taptapsee, camfind, and talking goggles object identification apps for the iphone,” AFB AccessWorld Magazine, 14, 2013. Web (CrossRef Link) Accessed: 2020-02-02.

[67] iDentifi: Mobile application for partially sighted and visually impaired people. Web (CrossRef Link). Accessed: 2020-02-02.

[68] Seeing AI: Talking camera app for those with a visual impairment. Retrieved September 15. Web (CrossRef Link) Accessed: 2020-02-02.

[69] BeSpecular: Let blind people see through your eyes. Web (CrossRef Link). Accessed: 2020-02-02.

[70] Microsoft Soundscape: A map delivered in 3D sound. Web (CrossRef Link). Accessed: 2020-02-02.

[71] Ahmetovic, D., Gleason, C., Ruan, C., Kitani, K., Takagi, H., Asakawa, C., “NavCog : a navigational cognitive assistant for the blind," in Proc. of the 18th International Conference on Human-Computer Interaction with Mobile Devices and Services, pp. 90-99, 2016. Article (CrossRef Link)

[72] Sato, D., Oh, U., Naito, K., Takagi, H., Kitani, K., Asakawa, C., "NavCog3 : An evaluation of a smartphone-based blind indoor navigation assistant with semantic features in a large-scale environment," in Proc. of the 19th International ACM SIGACCESS Conference on Computers and Accessibility, pp. 270-279, 2017. Article (CrossRef Link)

[73] G.I., Technologies(G3ict), C. Wayfindr., "Accelerating the Adoption of Indoor Audio Navigation with a Standard User Interface,” 2017. Article (CrossRef Link)

[74] WayFindr: Indoor Audio Wayfinding and Audio Navigation. Web (CrossRef Link). Accessed: 2020-02-02. 
[75] Intersection Explorer: Intersection Explorer helps blind users explore neighborhoods. Web (CrossRef Link). Accessed: 2020-02-02.

[76] Yatani, K., Banovic, N., Truong, K., "SpaceSense: representing geographical information to visually impaired people using spatial tactile feedback," in Proc. of the SIGCHI Conference on Human Factors in Computing Systems, pp. 415-424, 2012. Article (CrossRef Link)

[77] Blindsquare: Pioneering accessible navigation - indoors and outdoors. Web (CrossRef Link). Accessed: 2020-02-02.

[78] E.T. App Turns Smartphone Into Virtual Cane for the Blind, arXiv, Web (CrossRef Link). Accessed: 2020-02-02.

[79] Lazarillo: Orientation and autonomy for people with visual disability. Web (CrossRef Link). Accessed: 2020-02-02.

[80] Lazzus: Navigate the city and explore your surroundings independently. Web (CrossRef Link). Accessed: 2020-02-02.

[81] NaviLens: A brand new system of bidimensional, or BIDI, codes. Web (CrossRef Link). Accessed: 2020-02-02.

[82] SunuBand: It is your world. Explore it with the Sunu Band. Web (CrossRef Link). Accessed: 2020-02-02.

[83] OrCam MyEye 2: The most advanced wearable assistive technology for the blind and visually impaired, provides independence by conveying visual information, audibly. Web (CrossRef Link). Accessed: 2020-02-02.

[84] Toyota Project BLAID: Wearable technology to help the blind and visually impaired. Web (CrossRef Link). Accessed: 2020-02-02.

[85] eSight: We make glasses that let the visually impaired see. Web (CrossRef Link). Accessed: 2020-02-02.

[86] iBionics Diamond Eye: How a local startup is building a better bionic eye. Web (CrossRef Link). Accessed: 2020-02-02.

[87] Finn, A.P., Tripp, F., Whitaker, D., Vajzovic, L., "Synergistic visual gains attained using Argus II retinal prosthesis with OrCam MyEye,” Ophthalmology Retina, 2, 382-384, 2018.

Article (CrossRef Link) 


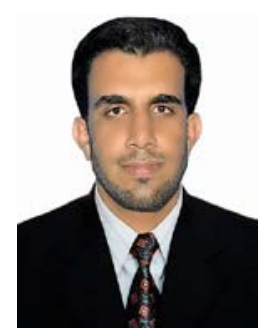

Waqas Nawaz received his Computer Science PhD degree from Kyung Hee University (South Korea) in 2015. He worked as a Post-Doctoral Fellow in the Institute of Information Systems, Innopolis University, Russia from 2015 to 2016. Afterwards, he joined Faculty of Computer and Information Systems, Islamic University of Madinah, KSA as Assistant Professor. His research interests include graph mining, social network analysis, databases, data mining, big data, image processing, and artificial intelligence.

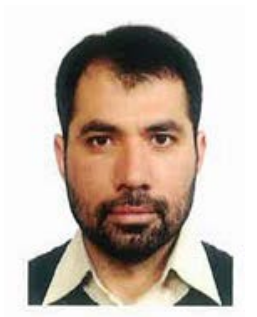

Kifayat Ullah Khan is working as Associate Professor in Department of Computer Science, National University of Computer and Emerging Sciences (FAST-NUCES), Islamabad, Pakistan. He received his Ph.D. degree from Kyung Hee University, Republic of Korea in 2016. He worked as a Post-Doctoral Fellow in the Department of Computer Science and Engineering at Kyung Hee University, South Korea from 2016 to 2018. His research interests are in Data Mining, Data Warehousing, Social Networks Analysis and Big Data.

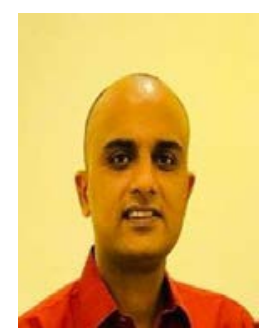

Khalid Bashir has a PhD in Computer Vision from Queen Mary, UoL, UK. He is a highly focused expert with the ability to drive research and academic results and needs. He is a highly cited and published scholar. He has specialized his research and teaching in machine learning, computer vision and deep learning. 